Supporting Information for

\title{
Strong Dual-Component Bioadhesive Based on Solventless Thiol-isocyanate Click Chemistry
}

Yifan Zhang, Xiaojie Li*, Wei Wei, and Xiaoya Liu*

Key Laboratory of Synthetic and Biological Colloids, Ministry of Education, School of Chemical and Material Engineering, Jiangnan University, No 1800 Lihu Avenue, Wuxi, Jiangsu, 214122, P. R. China.

*Correspondence to: xjli@jiangnan.edu.cn,lxy@jiangnan.edu.cn 


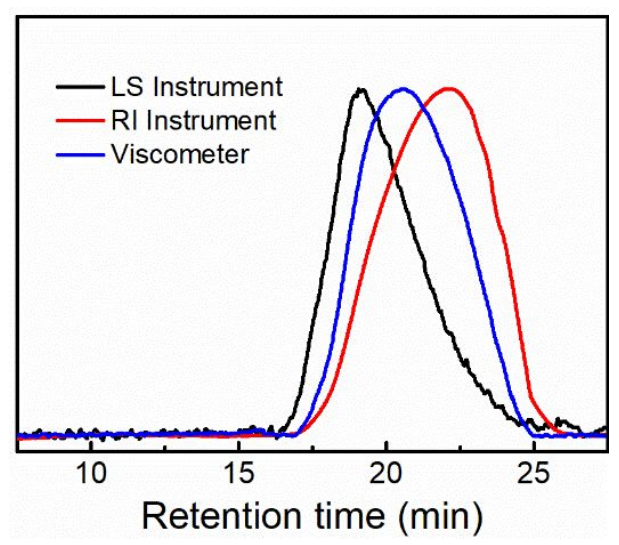

Figure S1: The molecular weight distribution curves of HBPTE recorded by light scattering (LS) detector, refractive index (RI) and viscometer, respectively.

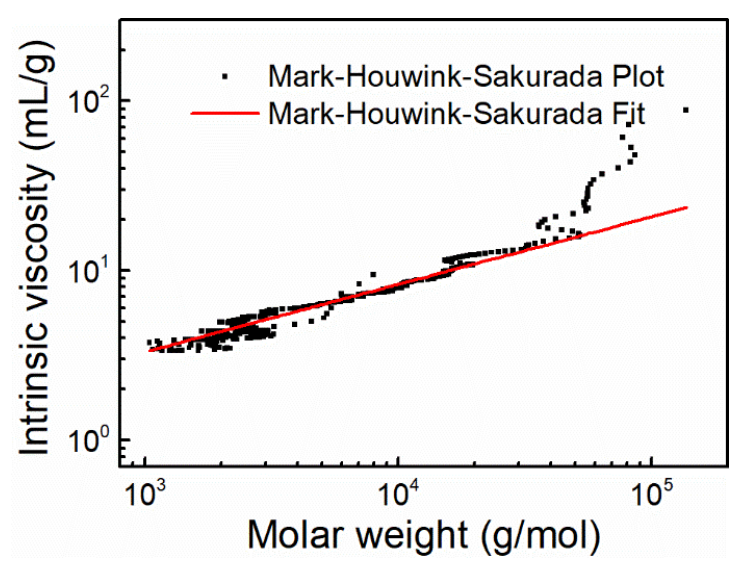

Figure S2: The Mark-Houwink-Sakurada plot and its fit line of hyperbranched polythioether.

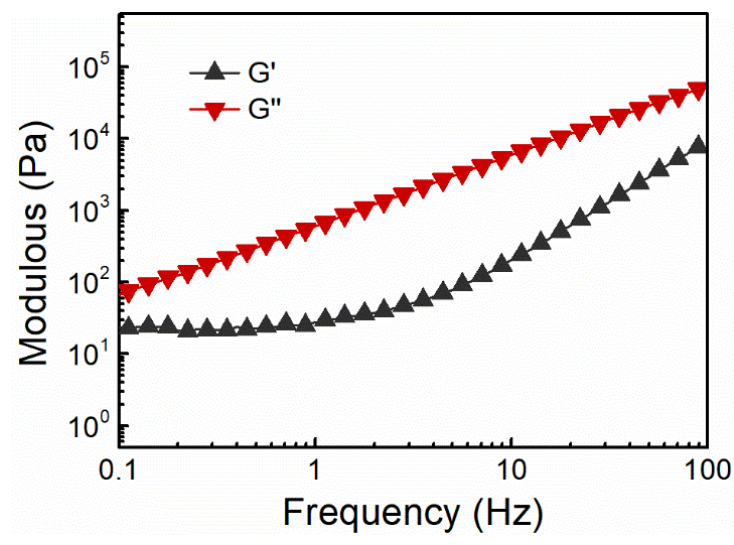

Figure S3: The dynamic modulus of $\mathrm{HBPTE}$ at $25^{\circ} \mathrm{C}$ as a function of frequency via rheological measurement. 


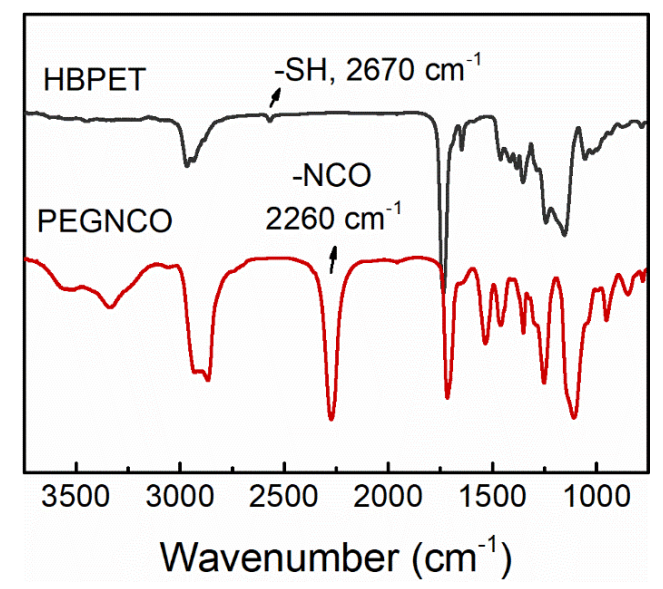

Figure S4: The FTIR spectras of hyperbranched polythioether and isocyanate modified polyethylene glycol.

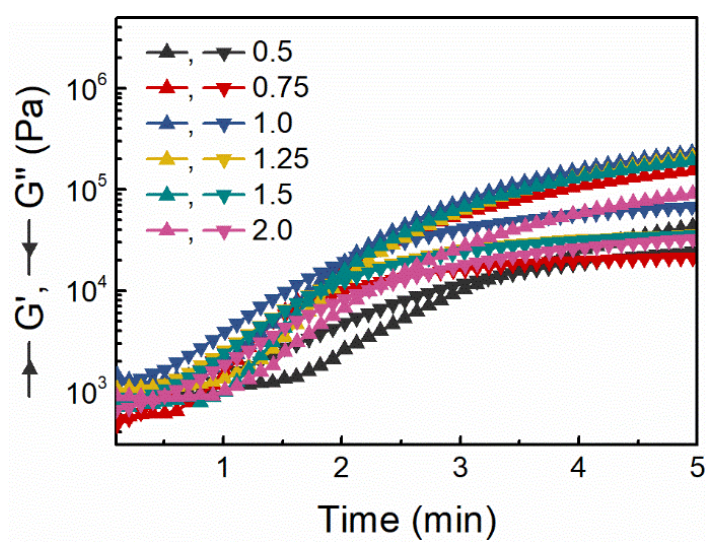

Figure S5: The real time rheological curve of HBPET-PEGNCO adhesive.
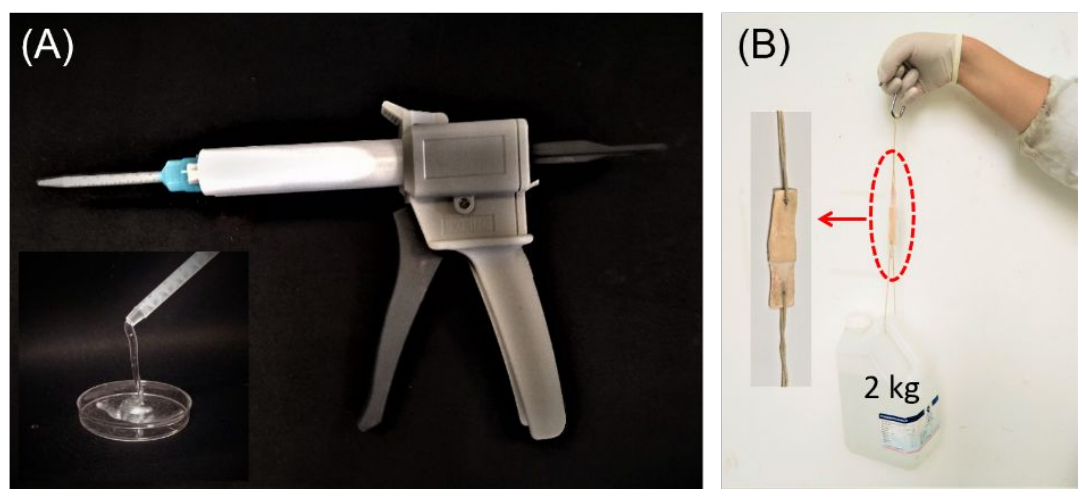

Figure S6: (A) The administration strategy using a commercial glue gun. (B) The A-1.0 bonded porcine skin with a $2 \mathrm{~cm} \times 2 \mathrm{~cm}$ adhesion area can lift a $2 \mathrm{~kg}$ weight. 


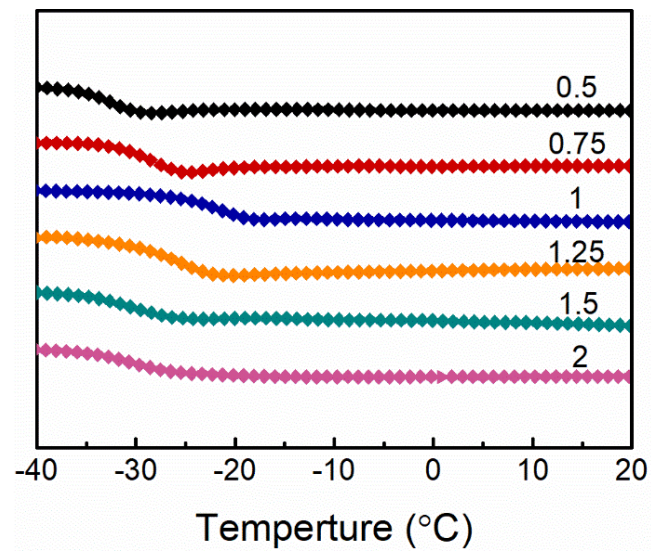

Figure S7: The DSC curves of cured HBPET-PEGNCO adhesives.

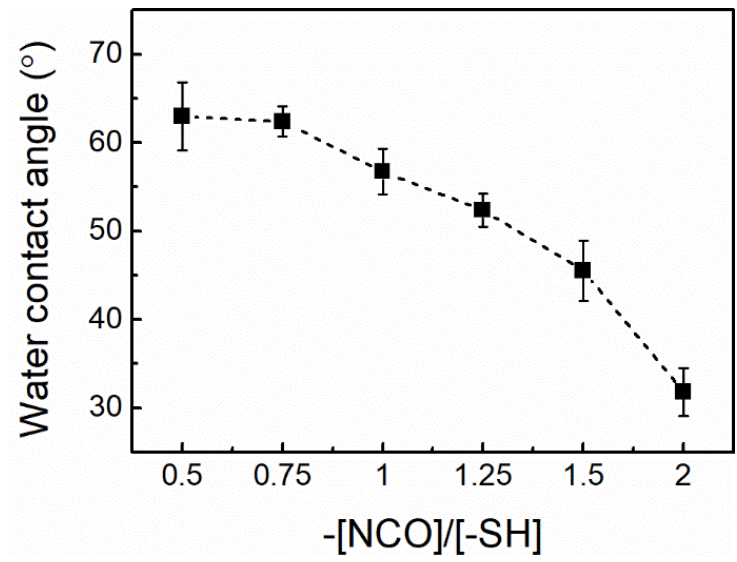

Figure S8: The water contact angle of cured HBPET-PEGNCO adhesive as a function of [$\mathrm{NCO}] /[-\mathrm{SH}]$ molar ratio.

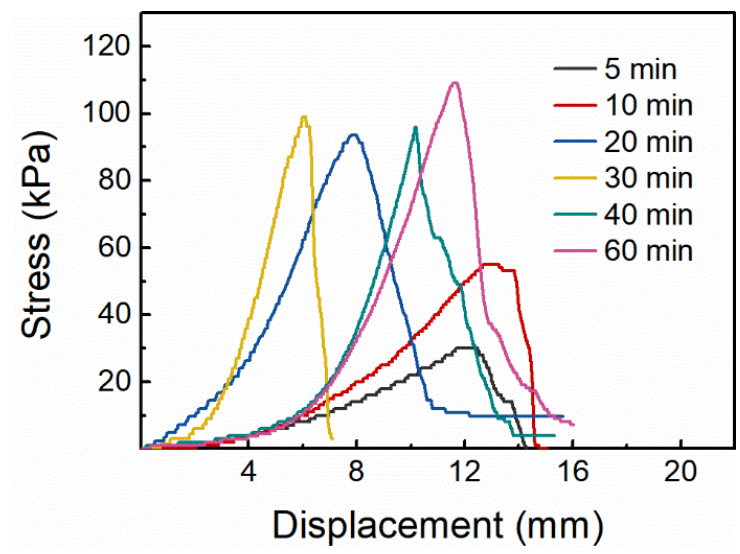

Figure S9: The lap-shear stress-displacement curves of A-1.0 to porcine skin at different bonding times. 


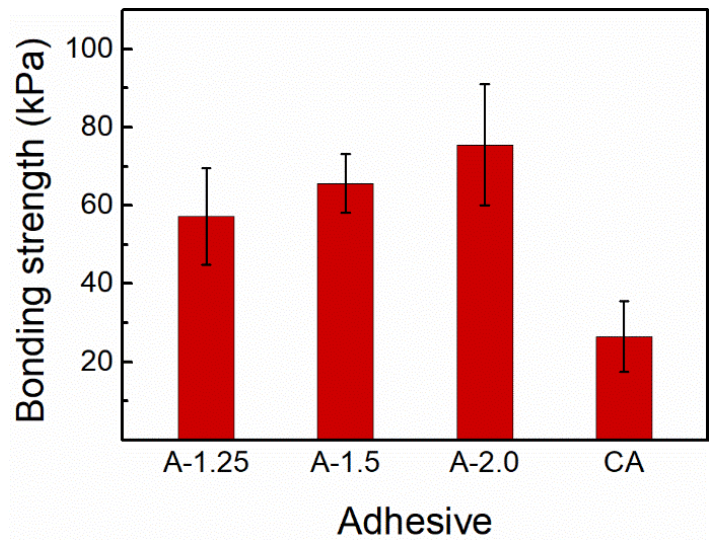

Figure S10: Bonding strengths of A-1.25, A-1.5, A-2.0 and cyanoacrylate glue on porcine skin containing in blood with a adhesion time of $1 \mathrm{~h}$.

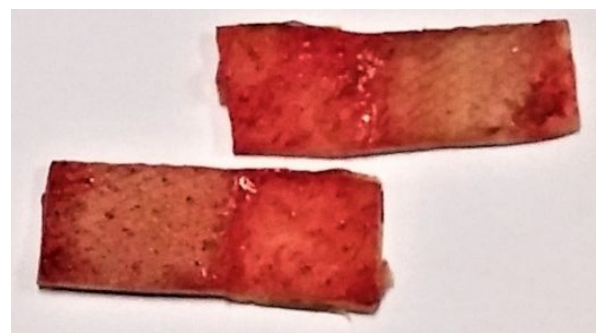

Figure S11: The photograph of porcine skin after bonding strengths measurement.

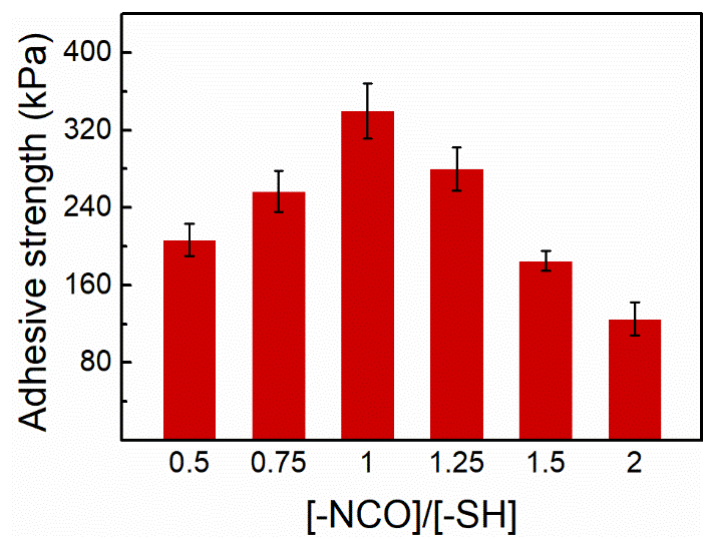

Figure S12: The lap-shear adhesive strength of HBPET-PEGNCO adhesives on stainless steel. 


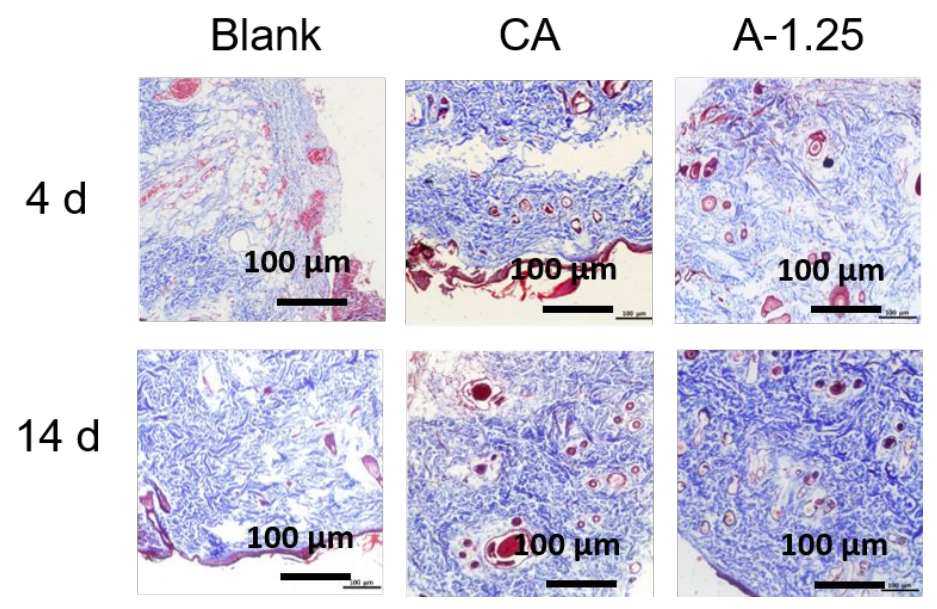

Figure S13: Masson's Trichrome staining of wounds after 14 days post-surgery treated with saline (control), cyanoacrylate glue, and A-1.25.

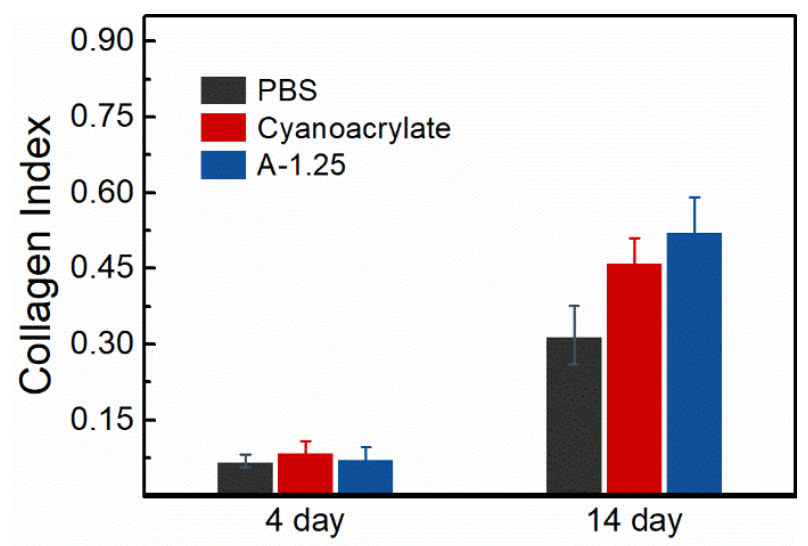

Figure S14: The relatively collagen index of wounds tissue after 14 days post-surgery treated with PBS (control), cyanoacrylate glue, and A-1.25. 